\title{
Umbilical cord blood mononuclear cell transplantation for neonatal hypoxic-ischemic encephalopathy
}

\author{
Pedro M. Pimentel-Coelho' ${ }^{1}$ Paulo H. Rosado-de-Castro ${ }^{2}$, Lea M. Barbosa da Fonseca ${ }^{2}$ and Rosalia Mendez-Otero'
}

Despite recent advances in the treatment of neonatal hypoxic-ischemic encephalopathy (HIE) using therapeutic hypothermia, at least $30 \%$ of the cooled infants will die or have moderate/severe neurological disability. Umbilical cord blood cells (UCBCs), which are readily available at birth, have been shown to reduce sensorimotor and/or cognitive impairments in several models of brain damage, representing a promising option for the treatment of neurological diseases. In this review, we discuss recent preclinical studies that assessed the effects of UCBC transplantation in the Rice-Vannucci animal model of HIE. We also review the possible cell types and mechanisms involved in the therapeutic effect of UCBC transplantation, including neuroprotection, immunomodulation, and stimulation of neural plasticity and regeneration. In addition, we discuss how neuroimaging methods, such as bioluminescence imaging, nuclear-medicine imaging, or magnetic resonance imaging, could be used to evaluate the biodistribution of UCBCs in both preclinical and clinical studies.

N eonatal encephalopathy is a clinical syndrome manifested by neurological symptoms in the first days of life in term infants. Several etiologies, including infections and metabolic and genetic disorders, can cause neonatal encephalopathy. However, when it is caused by perinatal asphyxia, as occurs in $30-60 \%$ of cases, the syndrome is called neonatal hypoxicischemic encephalopathy (HIE), which has an incidence of 1.5 per 1,000 live births (1).

HIE is one of the most important causes of moderate and severe neurological disability in children. Furthermore, longterm evaluations have found subtle cognitive deficits and alterations in daily-life behavioral functioning, even in cases of mild HIE (2).

Acutely evolving lesions can be observed in magnetic resonance imaging (MRI) scans of $80 \%$ of infants with neonatal encephalopathy and evidence of perinatal asphyxia, indicating that most of the lesions are acquired in the perinatal period and suggesting that a neuroprotective therapy could be achievable in the first hours after birth (3). Accordingly, recent clinical trials demonstrated the efficacy of therapeutic hypothermia when started within $6 \mathrm{~h}$ after birth, providing the first evidence that neuroprotection is feasible in newborns with HIE (4).

The therapeutic window of hypothermia coincides with a latent phase, when cerebral energy metabolism returns to normal following perinatal asphyxia. Using phosphorus magnetic resonance spectroscopy, it was demonstrated that brain energy metabolism returns to normal levels after a successful resuscitation, followed by a secondary energy failure after 6-24h. Many mechanisms that lead to secondary brain injury are already going on in the latent phase, including inflammation, production of nitric oxide/reactive oxygen species, glutamate excitotoxicity, and trophic factors withdrawal. All these mechanisms will result in mitochondrial permeabilization and cell death through activation of both caspase-dependent and -independent pathways $(4,5)$. Therefore, the existence of delayed and secondary neuronal death indicates that a subpopulation of neurons might be protected even if efficient neuroprotective treatments are initiated a few days after the hypoxic-ischemic (HI) insult.

Although therapeutic hypothermia is becoming a standard therapy for HIE, there is still a large number of infants who would benefit from additional therapies. For instance, one randomized study reported that therapeutic hypothermia reduced the incidence of disabling cerebral palsy from 30 to $19 \%$ and that the percentage of children with moderate HIE that died or developed moderate/severe disability was reduced from 48 to $32 \%$ (6). Hence, new treatments that might increase the benefits provided by this therapy are sorely needed. In this review, we will discuss the potential therapeutic role of umbilical cord blood cells (UCBCs) in HIE, which are readily available at birth and represent a promising option for the treatment of neurological disorders.

\section{HUMAN UMBILICAL CORD BLOOD (UCB)}

Public and private cord blood banks can store UCB units collected in utero or from the delivered placenta. UCB units stored in public banks are used for unrelated hematopoietic transplantation (after myeloablation) in children and adults with hematological malignancies and in children with other 
disorders, such as hemoglobinopathies, immunodeficiencies, and inborn errors of metabolism. When stored in private banks, UCBCs can be used for the treatment of biological siblings or for autologous transplantation in experimental studies. Autologous intravenous UCB transplantation is safe and feasible in young children with acquired neurological disorders (7) and is currently being evaluated in phase I/II clinical trials for the treatment of children with traumatic brain injury, acquired hearing loss, and cerebral palsy (ClinicalTrials.gov identifiers NCT01251003, NCT01343394, NCT01072370, and NCT01147653, respectively). Furthermore, the safety and feasibility of UCBC autologous transplantation are being evaluated in children with HIE, up to 14 days after birth, in a clinical trial conducted at Duke University (ClinicalTrials. gov identifier NCT00593242). These studies are based on the findings of preclinical studies suggesting that mononuclear UCBCs have a beneficial effect in several models of brain injury.

\section{Main Cell Types Present in the UCB}

To date, most of the preclinical studies assessing the role of UCBCs in the damaged central nervous system (CNS) have transplanted the UCB mononuclear cell fraction, which can be isolated by a density gradient and stored for a long period at cryogenic temperatures. Although it is still not clear which cell type(s) contribute(s) to the therapeutic effects of UCBC transplantation in neurological diseases, we will give an overview of the different cell populations present in the UCB mononuclear cell fraction and of the current data regarding their possible roles after a CNS injury.

Hematopoietic stem/progenitor cells (HPCs). Immediately after birth, the number of $\mathrm{CD} 34^{+}$HPCs in the peripheral blood of newborns is lower than in the UCB and decreases even more in the first $48 \mathrm{~h}$ postnatally (8). Moreover, UCB-derived CD $34^{+}$ HPCs have a less mature phenotype and proliferate more in response to cytokines in vitro (9).

Neuroprotective and immunomodulatory effects of bone marrow-derived HPCs have been observed in animal models of stroke. Intravenous injection of HPCs reduces the number of microglial cells and infiltrating $\mathrm{T}$ lymphocytes in the ischemic hemisphere, decreasing the infarct size of the treated animals (10). Therefore, it is possible that UCB-derived HPCs are partially responsible for the therapeutic effects of UCB mononuclear cells in HIE that will be discussed further on.

Endothelial progenitors. Hematopoietic stem cells and endothelial progenitor cells derive from a common precursor cell called hemangioblast and express several cell surface molecules in common, such as CD34, KDR (also known as vascular endothelial growth factor (VEGF) receptor 2), and Tie2 (one of the angiopoietin receptors, whose signaling regulates angiogenesis). When UCB mononuclear cells are cultured on fibronectin-coated plates, they give rise to mature endothelial cells that incorporate into endothelial networks (11). Accordingly, UCB CD $34^{+}$cell transplantation induces neovascularization in a mouse model of stroke (12). However, freshly isolated UCB mononuclear cells fail to improve perfusion in a murine model of hindlimb ischemia. In this model, enhanced neovascularization and perfusion is only obtained if endothelial progenitors are expanded in vitro before transplantation (13).

Lymphocytes. The UCB contains a similar percentage of B lymphocytes and a reduced percentage of $\mathrm{T}$ lymphocytes compared with the adult peripheral blood. In addition, UCB-derived lymphocytes are phenotypically and functionally immature (14).

A subpopulation of $\mathrm{T}$ lymphocytes, the regulatory $\mathrm{T}$ cells (Tregs), has a potent role in the suppression of immune responses, maintaining tolerance to self-antigens and preventing the development of autoimmune diseases. Treg cell lines isolated from the peripheral blood of adults are not uniform and may contain a mix of conventional and regulatory $\mathrm{T}$ cells, in contrast to UBC-derived Treg cell lines, which are more uniform and easily obtained. Moreover, UCB-derived Tregs have the same potent suppressor cell phenotype and function as adult Tregs (15), although they present a higher expansion capacity (16). Given the potent anti-inflammatory activity of Tregs, it has been suggested that these cells exert a significant neuroprotective role after stroke through IL-10 release (17).

Monocytes. Human monocytes can be divided into at least two main subsets, based on the expression of the cell surface antigens CD14 and CD16. These two populations express different cell adhesion molecules and chemokine receptors and have different roles after infection, ischemia, or other types of injury. Whereas the "classic" $\mathrm{CD} 14^{\text {high }} \mathrm{CD} 16^{-}$monocytes are associated with death and/or a poor outcome after stroke, $\mathrm{CD} 14^{\mathrm{dim}} \mathrm{CD} 16^{+}$monocytes are associated with a better outcome and a smaller infarction size (18). Likewise, there is a subset of bone marrow-derived monocytes that infiltrates the injured spinal cord, contributing to recovery through a local anti-inflammatory role (19).

Although the phagocytic ability of UCB-derived monocytes is not different from that of adult monocytes (20), one study found 168 genes that are differentially expressed between UCBderived and adult monocytes in the early response to lipopolysaccharide (21). Yerkovich and colleagues observed that monocytes are the main producers of tumor necrosis factor- $\alpha$ (TNF- $\alpha$ ) and IL- 6 when UCB mononuclear cells are stimulated with lipopolysaccharide and that Toll-like receptor-4 expression is higher and sustained in UCB-derived monocytes compared with adult monocytes. Moreover, UCB mononuclear cells produce more IL- 6 in response to lipopolysaccharide than cells of the peripheral blood of children and adults (22). Interestingly, IL-6 is a neurotrophic cytokine and enhances the expansion of neural stem/progenitor cells (23).

Mesenchymal stem/progenitor cells (MSCs). MSCs comprise a cell population that can differentiate into specialized mesenchymal cells, such as osteoblasts, chondrocytes, and adipocytes. MSCs can be isolated from most of the tissues in the 


\section{Review | Pimentel-Coelho et al.}

body, where they reside in perivascular niches, and can also be obtained from 1 in 10 UCB samples, suggesting that only a small number of MSCs circulates in the UCB (24). Two critical factors are associated with a successful isolation of MSCs from UCB: a volume of at least $90 \mathrm{ml}$ of blood and an interval of less than $2 \mathrm{~h}$ between collection and the beginning of cell processing (25). Bone marrow-derived MSCs have potent immunomodulatory and neuroprotective effects, beside inducing regeneration after HIE (26). However, given the low numbers of MSCs in the UCB, these cells are probably not involved in the beneficial effects of this therapy.

\section{Possible Mechanisms of Action of UCBCs in CNS Diseases}

Neuroprotection. The neuroprotective effect of human UCBCs was observed in numerous studies. In vitro, UCBCs protect neurons from glutamate-induced apoptosis through activation of the prosurvival Akt signaling pathway (27) and protect neurons from oxygen-glucose deprivation in ex vivo organotypic slice cultures (28). In addition, UCB $\mathrm{CD} 133^{+}$cells decrease apoptosis and prevent the suppression of axonal growth in hypoxic organ cocultures of the cerebral cortex and spinal cord of 3-d-old rats (29). Interestingly, both the UCB CD133-depleted mononuclear fraction, which has a decreased amount of HPCs, and the $\mathrm{CD}_{133^{+}}$-enriched fraction induce a similar neuroprotective effect in hypoxic neuronal cultures compared with the complete mononuclear cell fraction, suggesting that multiple cell types may be responsible for this effect. Moreover, both the direct and the indirect cocultivation of UCB mononuclear cells with the hypoxic neuronal cultures induce the same degree of neuroprotection, indicating that soluble factors secreted by UCBCs are largely, although not exclusively, responsible for the observed effects (30).

Giving support to this hypothesis is the fact that freshly isolated UCB mononuclear cells express the transcripts of the neurotrophic factors brain-derived neurotrophic factor (BDNF), glial cell line-derived neurotrophic factor (GDNF), nerve growth factor (NGF), neurotrophin (NT)-3, and NT-5 in higher amounts than peripheral blood mononuclear cells (31). VEGF, BDNF, NT-4, NT-5, and several cytokines and chemokines can also be detected in the culture supernatant of UCBCs $(31,32)$. Among these factors, NGF, BDNF, GDNF, NT-3, and VEGF have a neuroprotective effect in experimental models of HIE (33-36). Furthermore, the combined action of some of these factors may have a synergistic effect, as demonstrated by one study in which combined treatment with BDNF and epidermal growth factor (EGF) promoted a better functional recovery after HIE than treatment with each growth factor alone (37). This additive effect of multiple trophic factors has also been observed in several in vitro studies. For instance, a combination of trophic factors was necessary to promote the survival of oligodendrocytes (38), to rescue photoreceptors in a model of retinal dystrophy (39), and to direct axonal growth more efficiently (40).

Beside the neuroprotective effect, trophic factors could improve the neurological function after HIE by multiple mechanisms. VEGF increases the generation of new neurons in the two major neurogenic regions of the postnatal brain: the hippocampal dentate gyrus and the subventricular zone (41), in addition to being known as a major factor required for angiogenesis and vascular homeostasis. VEGF has also an important role in neuronal plasticity, improving hippocampal-dependent contextual memory (42).

Similarly, BDNF has multiple functions in the developing CNS and could provide a therapeutic effect after HIE by several mechanisms. BDNF protects the brain after HIE, decreasing neuronal death through activation of the extracellular signalrelated protein kinase pathway (33). Moreover, BDNF might affect neurogenesis, increasing the endogenous regenerative response of the brain, and could facilitate synaptic function and increase neural plasticity after injury $(41,43)$.

BDNF could also modulate the function of glial cells, given that its receptor, TrkB, is expressed by astrocytes and microglia. Indeed, BDNF induces a sustained increase in intracellular $\mathrm{Ca}^{2+}$ in microglia, inhibiting the release of nitric oxide from microglial cells activated by interferon- $\gamma(44)$.

Therefore, UCBC transplantation could represent a promising way to overcome the pharmacological challenge related to the systemic delivery of multiple therapeutic trophic factors to the brain (43). This potential could be further increased by genetic manipulation of the transplanted cells, using a nonviral lipofection technique that could increase the expression and secretion of one or more of these soluble factors by UCBCs (45).

Finally, the neuroprotective effect of UCBCs was also proven in vivo in animal models of stroke (46) and HIE (47), as will be discussed later.

Effects of UCBCs on glial cells. UCBCs protect mature oligodendrocytes from oxygen-glucose deprivation in vitro (48) and from cerebral ischemia in vivo (49). However, whether UCBs would have the same effect on oligodendrocyte progenitors, which are highly susceptible to $\mathrm{HI}$ insults during brain development, remains unknown.

UCBCs also affect the survival and function of other glial cells in vitro, protecting astrocytes from a hypoxic insult and modulating the secretion of IL-10 and IL- 6 by these cells (50). Beside playing an important role in the formation of the glial scar, astrocytes interact with neurons, blood vessels, perivascular cells, and microglial cells in the neurovascular unit, regulating cerebral blood flow and metabolism. Moreover, a subset of astrocytes may also be vulnerable to brain ischemia, dying after HIE (51).

In addition, UCBC transplantation exerts an antiinflammatory effect after stroke, decreasing the infiltration of $B$ cells and the expression of proinflammatory cytokines in the ischemic brain (52). This effect may be caused in part by a modulation of microglial cell function.

Microglial cells have an important role after brain injury, eliciting an innate immune response that clears cellular debris and induces brain repair, orchestrating brain inflammation. Although it has been recently shown that the depletion of microglial cells exacerbates brain injury in an animal model of 
perinatal stroke (53), the role of microglia after HIE remains unclear. Given that microglial cells may adopt different phenotypes, depending on the stimuli to which they are exposed, it is possible that these cells might have both beneficial and detrimental effects, depending on the context and on the phenotype they acquire over the course of the injury. For instance, endotoxin-activated microglial cells acquire a proinflammatory phenotype in vitro, decreasing neurogenesis and oligodendrogenesis of neural progenitor cells (54) and triggering the death of oligodendrocyte progenitor cells (55) and neurons (56). In contrast, IL-4-activated microglia stimulates oligodendrogenesis (54) and has a neuroprotective effect in vitro (57). This dichotomy, defined as microglia/macrophage polarization, has been extensively studied recently. "Classically" activated (also called M1) microglia/macrophages are activated by $\mathrm{T}$ helper 1 cytokines, such as interferon- $\gamma$, which induce the production of proinflammatory cytokines and cytotoxic mediators by these cells. However, when activated by $\mathrm{T}$ helper 2 cytokines (such as IL-4) or by Treg-derived IL-10 and transforming growth factor- $\beta$, microglial cells/macrophages acquire an anti-inflammatory phenotype, called "alternatively activated" (or M2), which is involved in neuroprotection, tissue remodeling, and regeneration (58). Although it has been shown that UBC-derived MSCs induce the alternative activation of microglial cells in an animal model of Alzheimer's disease (59), whether UCB mononuclear cells would promote the same effect remains unknown.

UCBCs decrease microglial survival after hypoxia in vitro (60) and reduce microglial activation in the cerebral cortex after HIE in rats (47). Similarly, UCBC transplantation reduces the accumulation of microglial cells in the cerebral cortex, striatum, and white matter and decreases the number of activated amoeboid microglia after stroke in rats (61). Furthermore, a decrease in microglial activation was also demonstrated after UCBC transplantation in aged rats, correlating with an increase in neurogenesis in the hippocampus (62). In this regard, UCBCs also increase neurogenesis in the subventricular zone after stroke (12), suggesting that these cells may enhance the endogenous regenerative capacity of the brain.

\section{UCBC TRANSPLANTATION IN ANIMAL MODELS OF HIE}

Four separate groups have evaluated the effects of UCBC transplantation in HIE using the Rice-Vannucci animal model, which consists of the ligation of one of the common carotid arteries, followed by a period of systemic hypoxia ( $8 \%$ oxygen and remaining nitrogen) in postnatal $\mathrm{d} 7$ rats.

Meier and colleagues showed that an intraperitoneal administration of UCBCs $24 \mathrm{~h}$ after injury decreases the sensorimotor impairment of $\mathrm{HI}$ animals, as assessed by footprint analysis and the cylinder test. They also performed in vivo electrophysiological recordings, showing that $\mathrm{UCBC}$ treatment prevents the decrease in size of the cortical hindpaw representation and reduces the HI-induced hyperexcitability of the ipsilateral primary somatosensory cortex $(63,64)$. The volume of the lesion was not changed by the treatment, in accordance with another study in which UCBCs were intravenously transplanted $24 \mathrm{~h}$ after injury
(65). However, in the latter study the treatment resulted only in a trend toward an improved performance in motor and cognitive tasks.

In contrast, a neuroprotective effect of UCBC transplantation was demonstrated when the cells were intraperitoneally injected $3 \mathrm{~h}$ after injury. The treatment reduced caspase-3 activation and the number of degenerating neurons in the striatum, resulting in the preservation of two primitive neurological reflexes that are affected by HIE. In addition, an anti-inflammatory effect was evidenced by a decrease in the number of activated microglial cells in the ipsilateral cerebral cortex of the treated animals (47).

The beneficial effects of UCBC transplantation can be observed even when the cells are transplanted $7 \mathrm{~d}$ after the injury. The delayed treatment reduces the deficits in motor symmetry and motor coordination in the elevated body swing test and in the rotarod. It also enhances synaptic plasticity in the hippocampus and increases the levels of the neurotrophic factors NGF, GDNF, and BDNF in the brain. Furthermore, UCBC transplantation in combination with mannitol, a drug that permeabilizes the blood-brain barrier, promotes a better improvement in motor function and further increases the levels of neurotrophic factors in the brain. Given that mannitol does not increase the number of UCBCs in the host brain, the authors suggested that blood-brain barrier permeabilization increases the entry of endogenous or UCBC-derived neurotrophic factors in the brain (66).

Moreover, a low number of cells $\left(1.5 \times 10^{4}\right.$ cells; approximately $1.1 \times 10^{3}$ cells/g body wt) was transplanted in this study. Based on these observations and assuming an average weight of $3.2 \mathrm{~kg}$, a minimum dose of $3.5 \times 10^{6}$ mononuclear cells, which can be easily obtained from a UCB unit, would be necessary to treat a newborn with HIE. However, a dose-response curve still must be defined in future preclinical studies, as well as the time window for the treatment.

\section{Biodistribution of UCB-Transplanted Cells}

Several studies have shown that UCBCs can be induced to acquire a neuronal phenotype under certain culture conditions. Therefore, it was tempting to speculate that UCBCs could be a source of neural cells for cell-replacement therapies. However, despite the expression of neuronal markers, functional voltagegated sodium channels, required for action potentials, are not present (67) or are present only in a minority of the UCBCderived neuron-like cells in vitro (68). Moreover, there is no evidence of differentiation of the donor cells into neurons, oligodendrocytes, or astrocytes when an adherently growing population of UCBCs is injected into the lateral ventricle of rat embryos (69).

The homing of UCBCs to the HI brain after an intraperitoneal transplantation was demonstrated by Meier and colleagues. They showed that the engraftment of UCBCs in the brain depends on the chemokine stromal-derived factor-1 (70) and that the transplanted cells do not differentiate into neurons or astrocytes in the HI brain (63). Moreover, other studies failed to find a large number of cells in the HI brain 
after intraperitoneal (47) or intravenous transplantation (66), despite the functional benefits of the treatment.

Taken together, these studies suggest that UCBCs do not exert their action in the HI brain through the replacement of lost cells. Even multipotent stem cells with a broader differentiation potential, such as neural stem cells, may exert their therapeutic effects in the CNS through multiple mechanisms beside cell replacement. For instance, neural stem cell transplantation results in neuroprotection, reduction of glial scarring, immunomodulation, and stimulation of endogenous mechanisms of brain plasticity and regeneration through paracrine effects (26). A recent study has also shown that unrestricted somatic stem cells, a subpopulation of UCBCs that can be expanded in vitro and induced to differentiate into apparently functional neurons under specific culture conditions (68), promote functional locomotor recovery when transplanted in the vicinity of an acute traumatic spinal cord injury in rats. Interestingly, the beneficial effects occurred in the absence of neural differentiation of the transplanted cells (71).

However, it is still possible that UCBCs could differentiate into cells of the myeloid lineage. Human CD $34^{+}$UCBCs migrate to the brain and differentiate into microglial cells when intravenously transplanted after whole-body irradiation in mice (72). Moreover, donor UCBCs differentiated into perivascular and parenchymal microglia/macrophages in a 6-y-old patient who was treated with hematopoietic stem cell transplantation for a severe type of Hunter disease (73).

Beside stromal-derived factor-1, other chemokines may be involved in the homing of UCBCs to the brain. For instance, the chemokines monocyte chemoattractant protein-1 (MCP-1) and macrophage inflammatory protein-1a (MIP-1 $\alpha)$ are involved in the recruitment of UCBCs to ischemic brain extracts (74). SDF-1, as well as MIP-1 $\alpha$ and MCP-1, is upregulated after HIE. MIP-1 $\alpha$ upregulation can be observed as early as $4 \mathrm{~h}$ after $\mathrm{HI}$, persisting for at least $5 \mathrm{~d}$ (75), whereas MCP-1 upregulation occurs from 2.5 to at least $48 \mathrm{~h}$ after HIE (76) and SDF-1 expression increases from $24 \mathrm{~h}$ to $7 \mathrm{~d}$ after injury (77). Furthermore, UCB mononuclear cells express the receptors for the neurotransmitter $\gamma$-aminobutyric acid and migrate in response to a gradient of $\gamma$-aminobutyric acid (78). Therefore, given the increased expression of chemoattractants in the HI brain, it remains unclear why UCBCs do not migrate massively to the brain after systemic transplantation. It is possible that UCBCs could be trapped in the lungs after the pulmonary passage (79), that they do not survive in the brain for a long time, and/or that these cells migrate to other organs such as the spleen. Indeed, UCBC transplantation has a systemic immunomodulatory effect after stroke, decreasing TNF- $\alpha$ and increasing IL-10 mRNA expression in the spleen. The treatment also reduces the stroke-induced alterations in spleen weight and $\mathrm{T}$ splenocyte proliferation (46).

Alternatively, an intra-arterial transplantation could increase the number of cells reaching the brain. A larger number of neural stem cells reached the brain after intra-arterial than after intravenous transplantation in an adult model of cerebral hypoxia-ischemia (80) and bone marrow-derived mononuclear cells persisted in the ischemic hemisphere for up to $48 \mathrm{~h}$ after an intra-arterial transplantation in a patient with stroke (81). This approach, beside being less invasive than an intracerebral transplantation, could also permit the combination of both the systemic effects and the local action of UCBCs in the CNS.

Therefore, it will be important to address the biodistribution of UCBCs after systemic transplantation in HIE to further understand and improve the therapeutic role of these cells. In this regard, noninvasive in vivo imaging of the injected cells may improve comprehension of several unsolved questions, including (i) the determination of the effectiveness of different methods of cell transplantation regarding cell migration and proliferation; (ii) the assessment of structural tissue recovery or adverse reactions; and (iii) the evaluation of dosing schedule and the number of cells to be transplanted.

\section{UCBC LABELING AND NEUROIMAGING METHODS}

One approach for the tracking of UCBCs is the transfection of reporter genes for bioluminescence imaging, nuclear-medicine imaging, or MRI. This approach is extremely valuable because it only reveals the activity of labeled cells that are viable and metabolically active. Moreover, stable transfection of cells allows long-term expression of the reporter gene, avoiding the problem seen with exogenous contrasts that are diluted with cell proliferation (82). Bioluminescence imaging has been successfully used to investigate the distribution of labeled cells in experimental models of neonatal and adult hypoxia-ischemia up to 30 days after injection $(80,83)$. However, although bioluminescence imaging remains an extremely important technique to allow clinical translation of experimental studies of cell therapies, it has limited spatial resolution $(2-3 \mathrm{~mm})$ and depth penetration $(1 \mathrm{~cm})$ and cannot be used in the clinic (82).

Similarly, in nuclear medicine a specific interaction between reporter gene product and an administered probe may be used to generate a signal that can be detected by modalities such as single-photon emission computed tomography (SPECT) and positron emission tomography (PET) (82). In an example, a recent human trial described that cytolytic $\mathrm{T}$ cells transduced with a herpes simplex virus type 1 thymidine kinase could be detected by PET scanning in the brain and in other parts of the body after intracranial infusion in patients with glioma (84). Transfection of genes may also be used to facilitate iron uptake by injected cells and therefore generate a signal that can be distinguished in MRI, precluding the need for exogenous contrast agents (85). Although these are promising techniques, further investigations are required to determine the safety of using viruses for transfection and the modification of intracellular iron pathways in neonatal diseases. Moreover, although the probability of developing cancer as a result of exposure to a single nuclear-medicine study is low (86), the use of reporter genes for PET and SPECT requiring multiple injections of radioactive probes would be a matter of concern regarding radiation exposure in the neonatal period.

A second approach is labeling cells with a radiopharmaceutical to be detected in nuclear medicine or an exogenous contrast to be detected in MRI. Radioisotope cell labeling has been used 
for decades to systemically monitor cells in nuclear-medicine studies such as leukocyte imaging to spot infections and has been used in children and premature infants (87). More recently, SPECT has been applied for monitoring cell therapies for stroke in animal models (88) and in humans (89) with radiopharmaceuticals such as technetium- $99 \mathrm{~m}\left({ }^{99 \mathrm{~m}} \mathrm{Tc}\right)$ and 111 -indium-oxine ( ${ }^{111}$ In-oxine). PET is already used for metabolism and neuroinflammation imaging in HIE patients (86) and has previously been used for cell labeling with ${ }^{18} \mathrm{~F}$-fluorodeoxyglycose (82). However, one of the greatest limitations of nuclear-medicine cell labeling seems to be the relatively short time window for imaging. ${ }^{99 \mathrm{~m}} \mathrm{Tc}$, the most frequently used radiopharmaceuti$\mathrm{cal}$, has a 6-h half-life, allowing imaging for only $24 \mathrm{~h}(87)$ and shows a low cytotoxicity profile $(87,90) .{ }^{111}$ In-oxine increases the cell-tracking window to $96 \mathrm{~h}$ but results in a lower resolution and higher radiation burden to the patient and to the transplanted cells (87). Several reports have indicated cell damage at $24-48 \mathrm{~h}$ postlabeling with ${ }^{111} \mathrm{In}$-oxine (91-94). Although ${ }^{99 \mathrm{~m}} \mathrm{Tc}$ carries a much lower radiation burden, studies have also suggested a decrease in the proliferative capacity that may be caused by radiation-induced DNA damage (95). Nevertheless, to our knowledge, there are currently no reports in the literature using cell labeling with radiopharmaceuticals in animal models of cell therapy for HIE.

Contrast agents for MRI may overcome many of the current labeling limitations of radiopharmaceuticals. Superparamagnetic iron oxide nanoparticles (SPIOs) such as ferumoxides (Feridex, Bayer Healthcare, NJ, and Endorem, Guerbet, France; 80-150 nm) and ferucarbotran (Resovist, Schering AG, Germany; 45-60 nm) were initially developed for intravenous injection to allow hepatic imaging and later adapted for cell labeling by numerous research groups. Although ferumoxides were recently taken off the market because of lack of sales for their original application, other clinical and nonclinical agents are constantly being developed (96). One advantage of SPIOs is that they are biodegradable and can be used by the cells in iron metabolism pathways. Moreover, they may be prepared with different properties such as core size (e.g., micrometer-size paramagnetic iron oxide particles), shape, and outer coating, which have the potential to modify their biologic activity (82). Techniques such as electroporation, or the incubation of SPIOs with non-clinical-grade unapproved lipofection transfection agents or with protamine sulfate (a drug approved by the US Food and Drug Administration) previous to cell labeling provide more efficient cell labeling $(82,96)$. Such labeled cells have been studied in animal models and imaged by preclinical and clinical MRI. Obenaus et al. (97) monitored the migration of SPIO-labeled murine neural stem cells after an intracerebral injection in an animal model of HIE, and Chen and colleagues (98) studied the distribution of MPIO-labeled MSCs in a rodent model of periventricular white matter injury in preterm infants.

Labeling of UCBCs with SPIOs for preclinical studies has also been performed by several groups and seems feasible (99-101). A labeling time of as low as $4 \mathrm{~h}$ was achieved without impairing viability and functional capability, an important step to allow rapid delivery of labeled cells in clinical studies (101).

In addition to animal studies, there have been reports on clinical trials that used SPIOs to label different cell types, such as neural stem cells (102), bone marrow mononuclear cells (103), MSCs (104), and UCBCs (105), with a follow-up of as long as 6 mo. In the latter study, the only study performed in a pediatric patient, intracerebroventricular injections of neurally committed human UCB-derived (NC-HUCB) cells were carried out in a 16-mo-old child, 7 mo after cardiac arrest-induced global hypoxia-ischemia (105). After $10 \mathrm{~d}$ in culture, cells were labeled with Feridex and grafted monthly by three serial injections into the lateral ventricle of the brain. MRI exams indicated neurally committed human UCB-derived cells extending along the lateral ventricle at $1 \mathrm{~d}$, $1 \mathrm{mo}$, and 2 mo after the injection, and the signal was scarcely visible at 4 and $6 \mathrm{mo}$. No clinical adverse events related to the cells were noted in any of the studies. However, SPIO labeling has limitations such as the aforementioned dilution of the contrast with cell division and the possibility that the iron from cells undergoing apoptosis or cell lysis may be internalized by tissue macrophages, leading to signal that could falsely be attributed to cells (106). Moreover, although many studies have found no adverse effects regarding cytotoxicity when labeling cells with SPIOs, recent studies have indicated that they may alter cellular events in MSCs (107-111). For instance, Chen et al. found that osteogenesis was inhibited by SPIOs, not because of cytotoxicity but instead by promoting cell mobilization (110). An alternative technique that has already been applied for imaging of UCBCs in animals is labeling with ${ }^{19} \mathrm{~F}$ MRI with liquid perfluorocarbon nanoparticles (112). The greatest advantage of this method seems to be that, unlike SPIO-based agents, it does not have measurable tissue background signal, which results in better contrastto-noise characteristics and allows definitive localization of the uniquely labeled cells. Moreover, it allows simultaneous tracking of multiple cell types, each carrying a unique MR signature, which is not possible with SPIO imaging and could be important for better identifying the complex roles and possible cooperative involvement of different cell populations used in regenerative therapies (112).

MRI is currently the preferred imaging modality to evaluate the pathogenesis, severity, and evolution of HIE (2). Therefore, the possibility of comparison of cell migration and techniques evaluating aspects such as infarct volume measuring and magnetic resonance spectroscopy will provide important information to future translation of cell therapies to the bedside.

\section{CONCLUSIONS}

UCBCs are available for autologous transplantation in the first few hours after birth, and recent preclinical studies have shown that UCB transplantation improves sensorimotor impairments after HIE (Figure 1). Although the mechanisms underlying this functional benefit are still poorly understood, it seems to involve a paracrine effect in which factors released by UCBCs contribute 

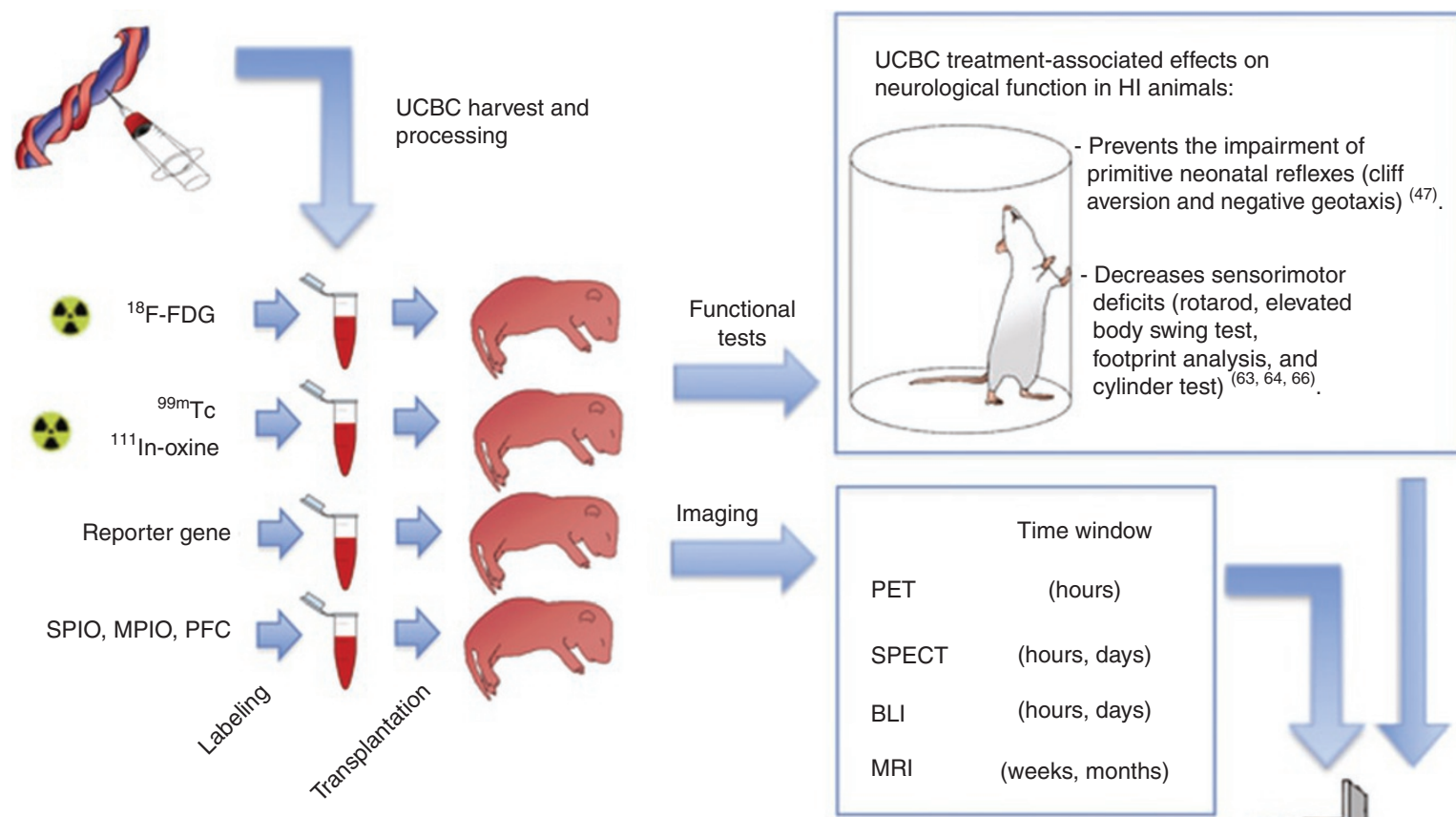

Beneficial effects of UCBC treatment in the HI brain:

- Decreases neuronal death in the striatum ${ }^{(47)}$;

- Decreases microglial activation in the cerebral cortex ${ }^{(47)}$;

- Increases the levels of GDNF, BDNF, and NGF in the brain ${ }^{(66)}$;

- Increases hippocampal CA1 dendritic density (66);

- Reduces neural processing impairments in the primary somatosensory cortex ${ }^{(64)}$.

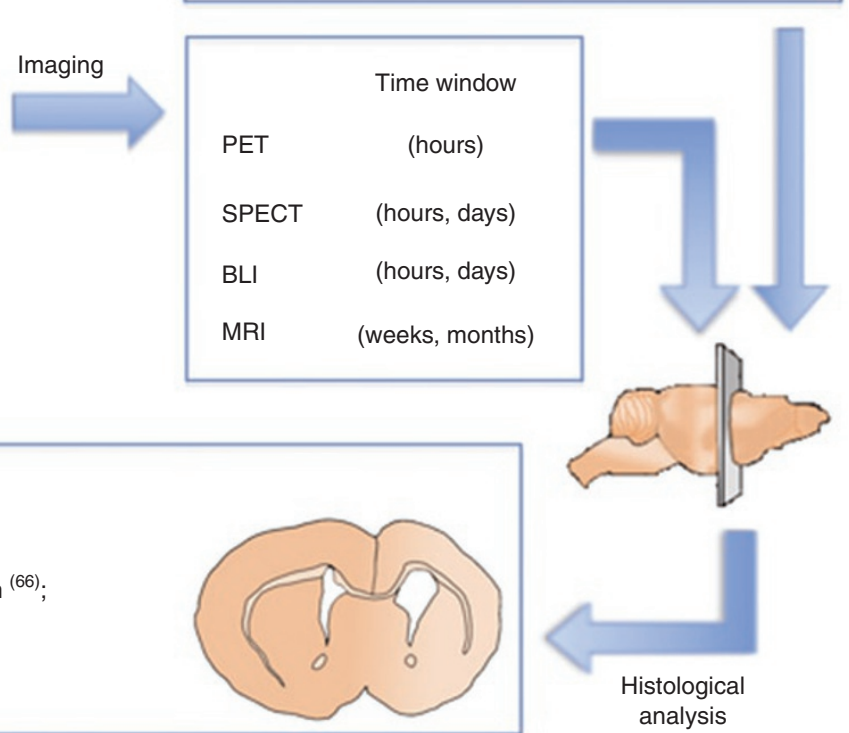

Figure 1. Schematic illustrating some of the possibilities for the assessment of cell therapies for neonatal hypoxic-ischemic encephalopathy (HIE). Umbilical cord blood cells (UCBCs) can be obtained from umbilical cord blood units collected in utero or from the delivered placenta. The biodistribution and the possible therapeutic effects of UCBC transplantation can be assessed in preclinical studies using animal models of HIE, as illustrated here. Cell tracking may be performed for hours with positron emission tomography (PET) using ${ }^{18} \mathrm{~F}$-fluorodeoxyglycose $\left({ }^{18} \mathrm{~F}-\mathrm{FDG}\right)$ or for hours to days with single-photon emission computed tomography (SPECT) using radiopharmaceuticals such as technetium-99m (99mTc) or 111-indium-oxine ( ${ }^{111}$ In-oxine). Another approach is to use reporter genes for bioluminescence imaging (BLI), which also allows cell monitoring for hours or days. On the other hand, magnetic resonance imaging (MRI) may permit increasing this time window for weeks to several months after transplantation when using contrasts such as superparamagnetic iron oxide (SPIO), micrometer-size paramagnetic iron oxide particles (MPIOs), or liquid perfluorocarbon nanoparticles (PFCs). Furthermore, the effects of UCBC transplantation on neurological function and the cellular and molecular mechanisms involved in the possible therapeutic effects can be evaluated by behavioral tests, biochemical analysis, histological analysis, and neuroimaging. The main findings observed after UCBC transplantation in the Rice-Vannucci animal model of HIE are summarized in the boxes. BDNF, brain-derived neurotrophic factor; GDNF, glial cell line-derived neurotrophic factor; $\mathrm{HI}$, hypoxic-ischemic; NGF, nerve growth factor.

to the decrease of neuronal death and the modulation of innate immune responses, reducing neural processing impairments and enhancing synaptic plasticity in the HI brain. Neuroimaging methods are valuable tools to evaluate the safety and the biodistribution of the cells over time, as well as the efficacy of the treatment in both preclinical (Figure 1) and clinical studies. Furthermore, we must understand the contribution of the different cell populations present in the UCB to the efficacy of the therapy and the time window more adequate for the treatment. Finally, it is still necessary to evaluate whether UCBCs transplantation could lead to a further improvement of neurological outcome when combined with therapeutic hypothermia.

\section{ACKNOWLEDGMENTS}

This work was supported by CNPq, CAPES, and FAPERJ. No competing financial interests exist.

\section{REFERENCES}

1. Kurinczuk JJ, White-Koning M, Badawi N. Epidemiology of neonatal encephalopathy and hypoxic-ischaemic encephalopathy. Early Hum Dev 2010;86:329-38.

2. de Vries LS, Jongmans MJ. Long-term outcome after neonatal hypoxic-ischaemic encephalopathy. Arch Dis Child Fetal Neonatal Ed 2010;95:F220-4.

3. Cowan F, Rutherford M, Groenendaal F, et al. Origin and timing of brain lesions in term infants with neonatal encephalopathy. Lancet 2003;361:736-42.

4. Johnston MV, Fatemi A, Wilson MA, Northington F. Treatment advances in neonatal neuroprotection and neurointensive care. Lancet Neurol 2011;10:372-82.

5. Hagberg H, Mallard C, Rousset CI, Xiaoyang Wang. Apoptotic mechanisms in the immature brain: involvement of mitochondria. J Child Neurol 2009;24:1141-6.

6. Shankaran S, Laptook AR, Ehrenkranz RA, et al. National Institute of Child Health and Human Development Neonatal Research Network. 
Whole-body hypothermia for neonates with hypoxic-ischemic encephalopathy. N Engl J Med 2005;353:1574-84.

7. Sun W, Buzanska L, Domanska-Janik K, Salvi RJ, Stachowiak MK. Voltage-sensitive and ligand-gated channels in differentiating neural stem-like cells derived from the nonhematopoietic fraction of human umbilical cord blood. Stem Cells 2005;23:931-45.

8. Kim JP, Lee YH, Lee YA, Kim YD. A comparison of the kinetics of nucleated cells and CD34+ cells in neonatal peripheral blood and cord blood. Biol Blood Marrow Transplant 2007;13:478-85.

9. Brown JA, Boussiotis VA. Umbilical cord blood transplantation: basic biology and clinical challenges to immune reconstitution. Clin Immunol 2008;127:286-97.

10. Schwarting S, Litwak S, Hao W, Bähr M, Weise J, Neumann H. Hematopoietic stem cells reduce postischemic inflammation and ameliorate ischemic brain injury. Stroke 2008;39:2867-75.

11. Murohara T. Therapeutic vasculogenesis using human cord blood-derived endothelial progenitors. Trends Cardiovasc Med 2001;11:303-7.

12. Taguchi A, Soma T, Tanaka H, et al. Administration of CD34+ cells after stroke enhances neurogenesis via angiogenesis in a mouse model. J Clin Invest 2004;114:330-8.

13. Finney MR, Greco NJ, Haynesworth SE, et al. Direct comparison of umbilical cord blood versus bone marrow-derived endothelial precursor cells in mediating neovascularization in response to vascular ischemia. Biol Blood Marrow Transplant 2006;12:585-93.

14. Harris DT, Schumacher MJ, Locascio J, et al. Phenotypic and functional immaturity of human umbilical cord blood T lymphocytes. Proc Natl Acad Sci USA 1992;89:10006-10.

15. Godfrey WR, Spoden DJ, Ge YG, et al. Cord blood CD4(+)CD25(+)derived $\mathrm{T}$ regulatory cell lines express FoxP3 protein and manifest potent suppressor function. Blood 2005;105:750-8.

16. Torelli GF, Maggio R, Peragine N, et al. Functional analysis and gene expression profile of umbilical cord blood regulatory T cells. Ann Hematol 2012;91:155-61.

17. Liesz A, Suri-Payer E, Veltkamp C, et al. Regulatory T cells are key cerebroprotective immunomodulators in acute experimental stroke. Nat Med 2009;15:192-9.

18. Urra X, Villamor N, Amaro S, et al. Monocyte subtypes predict clinical course and prognosis in human stroke. J Cereb Blood Flow Metab 2009;29:994-1002.

19. Shechter R, London A, Varol C, et al. Infiltrating blood-derived macrophages are vital cells playing an anti-inflammatory role in recovery from spinal cord injury in mice. PLoS Med 2009;6:e1000113.

20. Filias A, Theodorou GL, Mouzopoulou S, Varvarigou AA, Mantagos S, Karakantza M. Phagocytic ability of neutrophils and monocytes in neonates. BMC Pediatr 2011;11:29.

21. Lawrence S, Tang Y, Frank MB, et al. A dynamic model of gene expression in monocytes reveals differences in immediate/early response genes between adult and neonatal cells. J Inflamm (Lond) 2007;4:4.

22. Yerkovich ST, Wikström ME, Suriyaarachchi D, Prescott SL, Upham JW, Holt PG. Postnatal development of monocyte cytokine responses to bacterial lipopolysaccharide. Pediatr Res 2007;62:547-52.

23. Covey MV, Loporchio D, Buono KD, Levison SW. Opposite effect of inflammation on subventricular zone versus hippocampal precursors in brain injury. Ann Neurol 2011;70:616-26.

24. Secco M, Zucconi E, Vieira NM, et al. Multipotent stem cells from umbilical cord: cord is richer than blood! Stem Cells 2008;26:146-50.

25. Zhang X, Hirai M, Cantero S, et al. Isolation and characterization of mesenchymal stem cells from human umbilical cord blood: reevaluation of critical factors for successful isolation and high ability to proliferate and differentiate to chondrocytes as compared to mesenchymal stem cells from bone marrow and adipose tissue. J Cell Biochem 2011;112:1206-18.

26. Titomanlio L, Kavelaars A, Dalous J, et al. Stem cell therapy for neonatal brain injury: Perspectives and Challenges. Ann Neurol 2011;70:698-712.

27. Dasari VR, Veeravalli KK, Saving KL, et al. Neuroprotection by cord blood stem cells against glutamate-induced apoptosis is mediated by Akt pathway. Neurobiol Dis 2008;32:486-98.
28. Hall AA, Leonardo CC, Collier LA, Rowe DD, Willing AE, Pennypacker KR. Delayed treatments for stroke influence neuronal death in rat organotypic slice cultures subjected to oxygen glucose deprivation. Neuroscience 2009;164:470-7.

29. Tanaka N, Kamei N, Nakamae T, et al. CD133+ cells from human umbilical cord blood reduce cortical damage and promote axonal growth in neonatal rat organ co-cultures exposed to hypoxia. Int J Dev Neurosci 2010;28:581-7.

30. Reich DM, Hau S, Stahl T, et al. Neuronal hypoxia in vitro: investigation of therapeutic principles of HUCB-MNC and CD133+ stem cells. BMC Neurosci 2008;9:91.

31. Fan CG, Zhang QJ, Tang FW, Han ZB, Wang GS, Han ZC. Human umbilical cord blood cells express neurotrophic factors. Neurosci Lett 2005;380:322-5.

32. Newman MB, Willing AE, Manresa JJ, Sanberg CD, Sanberg PR. Cytokines produced by cultured human umbilical cord blood (HUCB) cells: implications for brain repair. Exp Neurol 2006;199:201-8.

33. Han BH, Holtzman DM. BDNF protects the neonatal brain from hypoxicischemic injury in vivo via the ERK pathway. J Neurosci 2000;20:5775-81.

34. Holtzman DM, Sheldon RA, Jaffe W, Cheng Y, Ferriero DM. Nerve growth factor protects the neonatal brain against hypoxic-ischemic injury. Ann Neurol 1996;39:114-22.

35. Galvin KA, Oorschot DE. Continuous low-dose treatment with brainderived neurotrophic factor or neurotrophin-3 protects striatal medium spiny neurons from mild neonatal hypoxia/ischemia: a stereological study. Neuroscience 2003;118:1023-32.

36. Feng Y, Rhodes PG, Bhatt AJ. Neuroprotective effects of vascular endothelial growth factor following hypoxic ischemic brain injury in neonatal rats. Pediatr Res 2008;64:370-4.

37. Im SH, Yu JH, Park ES, et al. Induction of striatal neurogenesis enhances functional recovery in an adult animal model of neonatal hypoxic-ischemic brain injury. Neuroscience 2010;169:259-68.

38. Barres BA, Schmid R, Sendnter M, Raff MC. Multiple extracellular signals are required for long-term oligodendrocyte survival. Development 1993;118:283-95.

39. Ogilvie JM, Speck JD, Lett JM. Growth factors in combination, but not individually, rescue rd mouse photoreceptors in organ culture. Exp Neurol 2000;161:676-85.

40. Cao X, Shoichet MS. Investigating the synergistic effect of combined neurotrophic factor concentration gradients to guide axonal growth. Neuroscience 2003;122:381-9.

41. Bath KG, Lee FS. Neurotrophic factor control of adult SVZ neurogenesis. Dev Neurobiol 2010;70:339-49.

42. Licht T, Goshen I, Avital A, et al. Reversible modulations of neuronal plasticity by VEGF. Proc Natl Acad Sci USA 2011;108:5081-6.

43. Nagahara AH, Tuszynski MH. Potential therapeutic uses of BDNF in neurological and psychiatric disorders. Nat Rev Drug Discov 2011;10:209-19.

44. Mizoguchi Y, Monji A, Kato T, et al. Brain-derived neurotrophic factor induces sustained elevation of intracellular $\mathrm{Ca} 2+$ in rodent microglia. J Immunol 2009;183:7778-86.

45. Yu G, Borlongan CV, Ou Y, et al. In vitro non-viral lipofectamine delivery of the gene for glial cell line-derived neurotrophic factor to human umbilical cord blood CD34+ cells. Brain Res 2010;1325:147-54.

46. Vendrame M, Gemma C, Pennypacker KR, et al. Cord blood rescues stroke-induced changes in splenocyte phenotype and function. Exp Neurol 2006;199:191-200.

47. Pimentel-Coelho PM, Magalhães ES, Lopes LM, deAzevedo LC, Santiago MF, Mendez-Otero R. Human cord blood transplantation in a neonatal rat model of hypoxic-ischemic brain damage: functional outcome related to neuroprotection in the striatum. Stem Cells Dev 2010;19:351-8.

48. Hall AA, Guyer AG, Leonardo CC, et al. Human umbilical cord blood cells directly suppress ischemic oligodendrocyte cell death. J Neurosci Res 2009;87:333-41.

49. Rowe DD, Leonardo CC, Hall AA, et al. Cord blood administration induces oligodendrocyte survival through alterations in gene expression. Brain Res 2010;1366:172-88. 
50. Jiang L, Womble T, Saporta S, et al. Human umbilical cord blood cells decrease microglial survival in vitro. Stem Cells Dev 2010;19: 221-8.

51. Villapol S, Gelot A, Renolleau S, Charriaut-Marlangue C. Astrocyte responses after neonatal ischemia: the yin and the yang. Neuroscientist 2008;14:339-44.

52. Vendrame M, Gemma C, de Mesquita D, et al. Anti-inflammatory effects of human cord blood cells in a rat model of stroke. Stem Cells Dev 2005;14:595-604.

53. Faustino JV, Wang X, Johnson CE, et al. Microglial cells contribute to endogenous brain defenses after acute neonatal focal stroke. J Neurosci 2011;31:12992-3001.

54. Butovsky O, Talpalar AE, Ben-Yaakov K, Schwartz M. Activation of microglia by aggregated beta-amyloid or lipopolysaccharide impairs MHC-II expression and renders them cytotoxic whereas IFN-gamma and IL-4 render them protective. Mol Cell Neurosci 2005;29:381-93.

55. Li J, Ramenaden ER, Peng J, Koito H, Volpe JJ, Rosenberg PA. Tumor necrosis factor alpha mediates lipopolysaccharide-induced microglial toxicity to developing oligodendrocytes when astrocytes are present. J Neurosci 2008;28:5321-30.

56. Lehnardt $\mathrm{S}$, Massillon L, Follett $\mathrm{P}$, et al. Activation of innate immunity in the CNS triggers neurodegeneration through a Toll-like receptor 4-dependent pathway. Proc Natl Acad Sci USA 2003;100:8514-9.

57. Butovsky O, Ziv Y, Schwartz A, et al. Microglia activated by IL-4 or IFNgamma differentially induce neurogenesis and oligodendrogenesis from adult stem/progenitor cells. Mol Cell Neurosci 2006;31:149-60.

58. Kigerl KA, Gensel JC, Ankeny DP, Alexander JK, Donnelly DJ, Popovich PG. Identification of two distinct macrophage subsets with divergent effects causing either neurotoxicity or regeneration in the injured mouse spinal cord. J Neurosci 2009;29:13435-44.

59. Lee JK, Jin HK, Endo S, Schuchman EH, Carter JE, Bae JS. Intracerebral transplantation of bone marrow-derived mesenchymal stem cells reduces amyloid-beta deposition and rescues memory deficits in Alzheimer's disease mice by modulation of immune responses. Stem Cells 2010;28:329-43.

60. Jiang L, Saporta S, Chen N, Sanberg CD, Sanberg P, Willing A. The effect of human umbilical cord blood cells on survival and cytokine production by post-ischemic astrocytes in vitro. Stem Cell Rev 2010;6:523-31.

61. Leonardo CC, Hall AA, Collier LA, Ajmo CT Jr, Willing AE, Pennypacker KR. Human umbilical cord blood cell therapy blocks the morphological change and recruitment of CD11b-expressing, isolectin-binding proinflammatory cells after middle cerebral artery occlusion. J Neurosci Res 2010;88:1213-22.

62. Bachstetter AD, Pabon MM, Cole MJ, et al. Peripheral injection of human umbilical cord blood stimulates neurogenesis in the aged rat brain. BMC Neurosci 2008;9:22.

63. Meier C, Middelanis J, Wasielewski B, et al. Spastic paresis after perinatal brain damage in rats is reduced by human cord blood mononuclear cells. Pediatr Res 2006;59:244-9.

64. Geissler M, Dinse HR, Neuhoff S, Kreikemeier K, Meier C. Human umbilical cord blood cells restore brain damage induced changes in rat somatosensory cortex. PLoS ONE 2011;6:e20194.

65. de Paula S, Vitola AS, Greggio S, et al. Hemispheric brain injury and behavioral deficits induced by severe neonatal hypoxia-ischemia in rats are not attenuated by intravenous administration of human umbilical cord blood cells. Pediatr Res 2009;65:631-5.

66. Yasuhara T, Hara K, Maki M, et al. Mannitol facilitates neurotrophic factor up-regulation and behavioural recovery in neonatal hypoxicischaemic rats with human umbilical cord blood grafts. J Cell Mol Med 2010;14:914-21.

67. Sun J, Allison J, McLaughlin C, et al. Differences in quality between privately and publicly banked umbilical cord blood units: a pilot study of autologous cord blood infusion in children with acquired neurologic disorders. Transfusion 2010;50:1980-7.

68. Greschat S, Schira J, Küry P, et al. Unrestricted somatic stem cells from human umbilical cord blood can be differentiated into neurons with a dopaminergic phenotype. Stem Cells Dev 2008;17:221-32.
69. Coenen M, Kögler G, Wernet P, Brüstle O. Transplantation of human umbilical cord blood-derived adherent progenitors into the developing rodent brain. J Neuropathol Exp Neurol 2005;64:681-8.

70. Rosenkranz K, Kumbruch S, Lebermann K, et al. The chemokine SDF-1/ CXCL12 contributes to the 'homing' of umbilical cord blood cells to a hypoxic-ischemic lesion in the rat brain. J Neurosci Res 2010;88:1223-33.

71. Schira J, Gasis M, Estrada V, et al. Significant clinical, neuropathological and behavioural recovery from acute spinal cord trauma by transplantation of a well-defined somatic stem cell from human umbilical cord blood. Brain 2011; e-pub ahead of print 8 September 2011.

72. Asheuer M, Pflumio F, Benhamida S, et al. Human CD34+ cells differentiate into microglia and express recombinant therapeutic protein. Proc Natl Acad Sci USA 2004;101:3557-62.

73. Araya K, Sakai N, Mohri I, et al. Localized donor cells in brain of a Hunter disease patient after cord blood stem cell transplantation. Mol Genet Metab 2009;98:255-63.

74. Jiang L, Newman M, Saporta S, et al. MIP-1alpha and MCP-1 induce migration of human umbilical cord blood cells in models of stroke. Curr Neurovasc Res 2008;5:118-24.

75. Cowell RM, Xu H, Galasso JM, Silverstein FS. Hypoxic-ischemic injury induces macrophage inflammatory protein-1alpha expression in immature rat brain. Stroke 2002;33:795-801.

76. Ivacko J, Szaflarski J, Malinak C, Flory C, Warren JS, Silverstein FS. Hypoxic-ischemic injury induces monocyte chemoattractant protein-1 expression in neonatal rat brain. J Cereb Blood Flow Metab 1997;17: 759-70.

77. Miller JT, Bartley JH, Wimborne HJ, et al. The neuroblast and angioblast chemotaxic factor SDF-1 (CXCL12) expression is briefly up regulated by reactive astrocytes in brain following neonatal hypoxic-ischemic injury. BMC Neurosci 2005;6:63.

78. Zangiacomi V, Balon N, Maddens S, Tiberghien P, Versaux-Botteri C, Deschaseaux F. Human cord blood-derived hematopoietic and neural-like stem/progenitor cells are attracted by the neurotransmitter GABA. Stem Cells Dev 2009;18:1369-78.

79. Fischer UM, Harting MT, Jimenez F, et al. Pulmonary passage is a major obstacle for intravenous stem cell delivery: the pulmonary first-pass effect. Stem Cells Dev 2009;18:683-92.

80. Pendharkar AV, Chua JY, Andres RH, et al. Biodistribution of neural stem cells after intravascular therapy for hypoxic-ischemia. Stroke 2010;41:2064-70.

81. Barbosa da Fonseca LM, Battistella V, de Freitas GR, et al. Early tissue distribution of bone marrow mononuclear cells after intra-arterial delivery in a patient with chronic stroke. Circulation 2009;120:539-41.

82. Arbab AS, Janic B, Haller J, Pawelczyk E, Liu W, Frank JA. In Vivo Cellular Imaging for Translational Medical Research. Curr Med Imaging Rev 2009;5:19-38.

83. Daadi MM, Davis AS, Arac A, et al. Human neural stem cell grafts modify microglial response and enhance axonal sprouting in neonatal hypoxicischemic brain injury. Stroke 2010;41:516-23.

84. Yaghoubi SS, Jensen MC, Satyamurthy N, et al. Noninvasive detection of therapeutic cytolytic T cells with 18F-FHBG PET in a patient with glioma. Nat Clin Pract Oncol 2009;6:53-8.

85. Genove G, DeMarco U, Xu H, Goins WF, Ahrens ET. A new transgene reporter for in vivo magnetic resonance imaging. Nat Med 2005;11: $450-4$.

86. Kannan S, Chugani HT. Applications of positron emission tomography in the newborn nursery. Semin Perinatol 2010;34:39-45.

87. Palestro CJ, Love C, Bhargava KK. Labeled leukocyte imaging: current status and future directions. Q J Nucl Med Mol Imaging 2009;53: 105-23.

88. Lappalainen RS, Narkilahti S, Huhtala T, et al. The SPECT imaging shows the accumulation of neural progenitor cells into internal organs after systemic administration in middle cerebral artery occlusion rats. Neurosci Lett 2008;440:246-50.

89. Barbosa da Fonseca LM, Gutfilen B, Rosado de Castro PH, et al. Migration and homing of bone-marrow mononuclear cells in chronic ischemic stroke after intra-arterial injection. Exp Neurol 2010;221:122-8. 
90. van Hemert FJ, Thurlings R, Dohmen SE, et al. Labeling of autologous monocytes with 99mTc-HMPAO at very high specific radioactivity. Nucl Med Biol 2007;34:933-8.

91. Brenner W, Aicher A, Eckey T, et al. 111In-labeled CD34+ hematopoietic progenitor cells in a rat myocardial infarction model. J Nucl Med 2004;45:512-8.

92. Chen MF, Lin CT, Chen WC, et al. The sensitivity of human mesenchymal stem cells to ionizing radiation. Int J Radiat Oncol Biol Phys 2006;66:244-53.

93. Nowak B, Weber C, Schober A, et al. Indium-111 oxine labelling affects the cellular integrity of haematopoietic progenitor cells. Eur J Nucl Med Mol Imaging 2007;34:715-21.

94. Gholamrezanezhad A, Mirpour S, Ardekani JM, et al. Cytotoxicity of 111In-oxine on mesenchymal stem cells: a time-dependent adverse effect. Nucl Med Commun 2009;30:210-6.

95. Pedraza-López M, Ferro-Flores G, Mendiola-Cruz MT, Morales-Ramírez P. Assessment of radiation-induced DNA damage caused by the incorporation of $99 \mathrm{mTc}$-radiopharmaceuticals in murine lymphocytes using single cell gel electrophoresis. Mutat Res 2000;465:139-44.

96. Bulte JW. In vivo MRI cell tracking: clinical studies. AJR Am J Roentgenol 2009;193:314-25.

97. Obenaus A, Dilmac N, Tone B, et al. Long-term magnetic resonance imaging of stem cells in neonatal ischemic injury. Ann Neurol 2011;69:282-91.

98. Chen A, Siow B, Blamire AM, Lako M, Clowry GJ. Transplantation of magnetically labeled mesenchymal stem cells in a model of perinatal brain injury. Stem Cell Res 2010;5:255-66.

99. Daldrup-Link HE, Rudelius M, Oostendorp RA, et al. Comparison of iron oxide labeling properties of hematopoietic progenitor cells from umbilical cord blood and from peripheral blood for subsequent in vivo tracking in a xenotransplant mouse model XXX. Acad Radiol 2005;12:502-10.

100. Arbab AS, Yocum GT, Rad AM, et al. Labeling of cells with ferumoxidesprotamine sulfate complexes does not inhibit function or differentiation capacity of hematopoietic or mesenchymal stem cells. NMR Biomed 2005; 18:553-9.

101. Janic B, Rad AM, Jordan EK, et al. Optimization and validation of FePro cell labeling method. PLoS ONE 2009;4:e5873.
102. Zhu J, Zhou L, XingWu F. Tracking neural stem cells in patients with brain trauma. N Engl J Med 2006;355:2376-8.

103. Callera F, de Melo CM. Magnetic resonance tracking of magnetically labeled autologous bone marrow CD34+ cells transplanted into the spinal cord via lumbar puncture technique in patients with chronic spinal cord injury: CD34+ cells' migration into the injured site. Stem Cells Dev 2007;16:461-6.

104. Karussis D, Karageorgiou C, Vaknin-Dembinsky A, et al. Safety and immunological effects of mesenchymal stem cell transplantation in patients with multiple sclerosis and amyotrophic lateral sclerosis. Arch Neurol 2010;67:1187-94.

105. Jozwiak S, Habich A, Kotulska K, et al. Intracerebroventricular transplantation of cord blood-derived neural progenitors in a child with severe global brain ischemic injury. Cell Medicine 2010;1:71-80.

106. Terrovitis J, Stuber M, Youssef A, et al. Magnetic resonance imaging overestimates ferumoxide-labeled stem cell survival after transplantation in the heart. Circulation 2008;117:1555-62.

107. Kostura L, Kraitchman DL, Mackay AM, Pittenger MF, Bulte JW. Feridex labeling of mesenchymal stem cells inhibits chondrogenesis but not adipogenesis or osteogenesis. NMR Biomed 2004;17:513-7.

108. Farrell E, Wielopolski P, Pavljasevic P, et al. Effects of iron oxide incorporation for long term cell tracking on MSC differentiation in vitro and in vivo. Biochem Biophys Res Commun 2008;369:1076-81.

109. Huang DM, Hsiao JK, Chen YC, et al. The promotion of human mesenchymal stem cell proliferation by superparamagnetic iron oxide nanoparticles. Biomaterials 2009;30:3645-51.

110. Chen YC, Hsiao JK, Liu HM, et al. The inhibitory effect of superparamagnetic iron oxide nanoparticle (Ferucarbotran) on osteogenic differentiation and its signaling mechanism in human mesenchymal stem cells. Toxicol Appl Pharmacol 2010;245:272-9.

111. Kim HS, Oh SY, Joo HJ, Son KR, Song IC, Moon WK. The effects of clinically used MRI contrast agents on the biological properties of human mesenchymal stem cells. NMR Biomed 2010;23:514-22.

112. Partlow KC, Chen J, Brant JA, et al. 19F magnetic resonance imaging for stem/progenitor cell tracking with multiple unique perfluorocarbon nanobeacons. FASEB J 2007;21:1647-54. 